\title{
Kaposi sarcoma herpesvirus-induced cellular reprogramming contributes to the lymphatic endothelial gene expression in Kaposi sarcoma
}

\author{
Hsei-Wei Wang ${ }^{1}$, Matthew W B Trotter ${ }^{1}$, Dimitrios Lagos ${ }^{1}$, Dimitra Bourboulia ${ }^{1}$, Stephen Henderson ${ }^{1}$, \\ Taija Mäkinen ${ }^{2}$, Stephen Elliman ${ }^{1}$, Adrienne M Flanagan ${ }^{3}$, Kari Alitalo $^{2} \&$ Chris Boshoff $^{1}$
}

The biology of Kaposi sarcoma is poorly understood because the dominant cell type in Kaposi sarcoma lesions is not known ${ }^{1-4}$. We show by gene expression microarrays that neoplastic cells of Kaposi sarcoma are closely related to lymphatic endothelial cells (LECs) and that Kaposi sarcoma herpesvirus (KSHV) ${ }^{5,6}$ infects both LECs and blood vascular endothelial cells (BECs) in vitro. The gene expression microarray profiles of infected LECs and BECs show that KSHV induces transcriptional reprogramming of both cell types. The lymphangiogenic molecules VEGF-D and angiopoietin-2 were elevated in the plasma of individuals with acquired immune deficiency syndrome and Kaposi sarcoma. These data show that the gene expression profile of Kaposi sarcoma resembles that of LECs, that KSHV induces a transcriptional drift in both LECs and BECs and that lymphangiogenic molecules are involved in the pathogenesis of Kaposi sarcoma.

The cellular origin of the spindle cells of Kaposi sarcoma lesions is poorly defined ${ }^{3,7}$. Kaposi sarcoma spindle cells express endothelial cell markers but also have features of other cell lineages, including fibroblasts, macrophages and smooth muscle cells ${ }^{1-3}$. Kaposi sarcoma could be a tumor originating from LECs, as spindle cells ubiquitously express VEGFR-3 and podoplanin and stain with the antibody D2-40 recognizing $\mathrm{LECs}^{4,8}$. But these markers can also be expressed on angiogenic blood vessels, or on other cell types ${ }^{9}$. Furthermore, some

Figure 1 GEM data for Kaposi sarcoma and cell groups. (a) A group of 5,894 genes normalized and filtered $(0.5<$ coefficient of variance $<10)$ using default parameters of the DChip package ${ }^{13}$ were used to construct a dendrogram representing relationships between the 35 samples. KS, Kaposi sarcoma. (b) A heat map of 1,482 genes that differentiate Kaposi sarcoma $(K S)$ and skin sample groups $(q \leq 0.05)$. Selected downregulated genes are shown in blue and upregulated genes in red. Cytokines and chemokines and their receptors are underlined. The full list of genes is available in Supplementary Table 1 online. Color scale indicates units of standard deviation (s.d.) from the mean expression of each gene.
BEC markers (e.g., CD34) are expressed in all Kaposi sarcoma spindle cells $^{1}$. KSHV is the infectious cause of Kaposi sarcoma ${ }^{5,6}$. In vitro, KSHV can infect both micro- and macrovascular endothelial cells, and these cells are useful to study the role of KSHV in the pathogenesis of Kaposi sarcoma ${ }^{10-12}$.

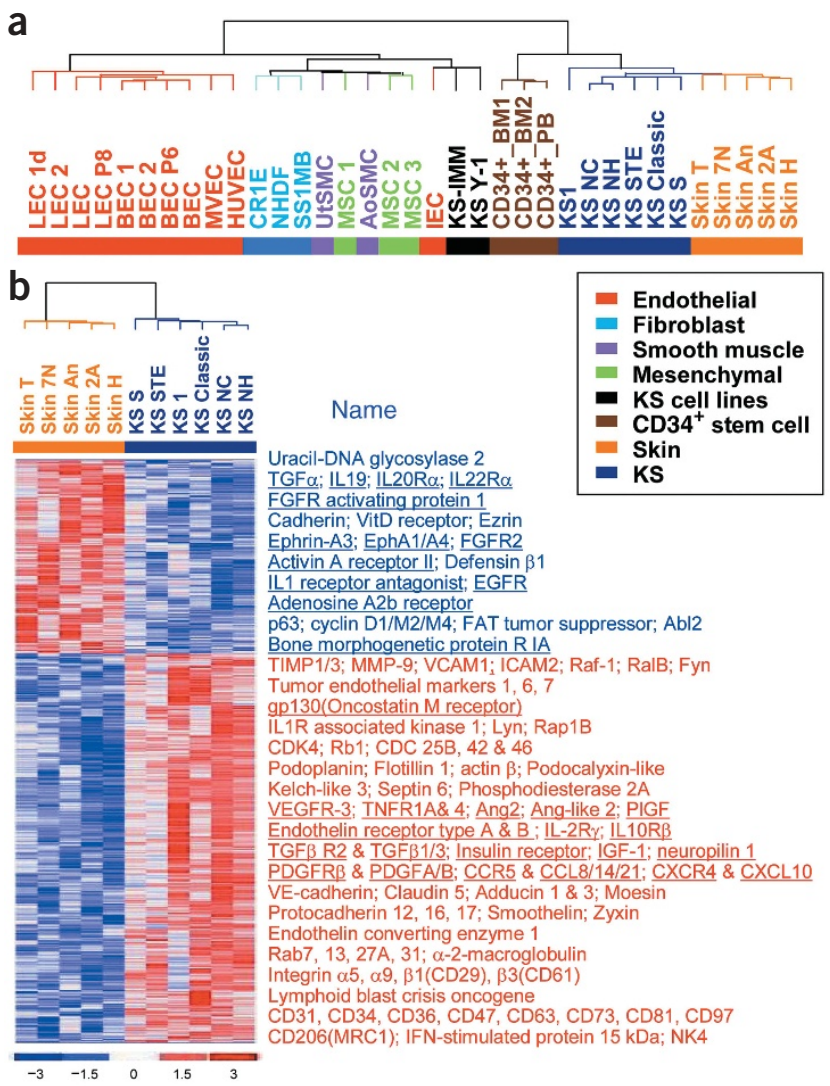

${ }^{1}$ Cancer Research UK, Viral Oncology Group, Wolfson Institute for Biomedical Research, Cruciform Building, Gower Street, University College London, London WC1E 6BT, UK. ${ }^{2}$ Molecular/Cancer Biology Laboratory and Ludwig Institute for Cancer Research, Biomedicum Helsinki and Helsinki University Central Hospital, University of Helsinki, Finland. ${ }^{3}$ Institute of Orthopaedics, University College London, UK. Correspondence should be addressed to C.B. (c.boshoff@ucl.ac.uk). 
We used oligonucleotide microarrays to compare the gene expression microarray (GEM) profile of samples of Kaposi sarcoma and normal skin to various cell cultures. We used nodular Kaposi sarcoma biopsy samples with $>80 \%$ spindle cells to minimize the dermal and epidermal components. Hierarchical clustering of 35 samples using 5,894 filtered genes ${ }^{13}$ showed that each group of samples was distinct (Fig. 1a). More genes are similarly overexpressed between Kaposi sarcoma and endothelial cells than between Kaposi sarcoma and any other cell type that we tested (Supplementary Fig. 1 online).

Hierarchical clustering showed that Kaposi sarcoma, skin and CD $34^{+}$cells clustered together, indicative of the relative abundance of CD $34^{+}$cells in both Kaposi sarcoma and skin. To eliminate the common tissue background present in normal skin biopsy samples, we selected a subset of the global expression profile to provide a 'Kaposi sarcoma expression signature' by removing genes that were expressed at a similar level in both Kaposi sarcoma and the dermis or epidermis. The signature contained 1,482 genes that differentiate Kaposi sarcoma from normal skin $(q \leq 0.05$; Fig. $\mathbf{1 b}$ and Supplementary Table 1 online). Despite the rich lymphatic network of normal skin, known LEC markers VEGFR-3, podoplanin and CD206 were present in the Kaposi sarcoma expression signature. We used Kaposi sarcoma biopsy samples, rather than cells cultured from primary lesions, to create the Kaposi sarcoma expression signature, because cells from primary lesions can represent many cell types, including fibroblasts and

Table 1 Cytokine network in Kaposi sarcoma

\begin{tabular}{|c|c|c|c|}
\hline Ligand & Ligand up & Receptor up & Receptor \\
\hline \multicolumn{4}{|l|}{ Paracrine } \\
\hline $\mathrm{TNF} \alpha$ & - & + & TNF-R1/R4 \\
\hline IL-6 & - & + & gp130 \\
\hline Oncostatin M & - & + & gp130 \\
\hline VEGF-C/VEGF-D & - & + & VEGFR3 \\
\hline Insulin & - & + & Insulin R \\
\hline IL-10 & - & + & IL-10R $\beta$ \\
\hline IL-2 & - & + & $\mathrm{IL}-2 \mathrm{R} \gamma$ \\
\hline Thrombospondin & - & + & CD36 \\
\hline VEGF165/VEGFB/PIGF-2 & - & + & Neuropilin 1 \\
\hline CXCL12 & - & + & CXCR4 \\
\hline \multicolumn{4}{|l|}{ Autocrine } \\
\hline PDGFA/PDGFB & + & + & PDGFR $\beta$ \\
\hline TGF $\beta 1 /$ TGF $\beta 3$ & + & + & $\operatorname{TGF} \beta$ R2 \\
\hline IGF-1 & + & $-^{*}$ & IGF-1R \\
\hline ANG2 & + & $-*$ & Tie-2 \\
\hline CCL5 (RANTES) & + & + & CCR5 \\
\hline CCL8 (MCP-2) & + & + & CCR5 \\
\hline Endothelin & + & + & Type A and B \\
\hline vIL6 & + & + & gp130 \\
\hline vMIP-I/vMIP-II/vMIP-III & + & + & CCR5/CXCR4 \\
\hline \multicolumn{4}{|l|}{ Unknown } \\
\hline Ang-like 2 & + & $?$ & $?$ \\
\hline Inhibin $\beta B$ & + & - & Inhibin R \\
\hline PIGF & + & - & VEGFR 1 \\
\hline CXCL10 & + & - & CXCR3 \\
\hline CCL14 (HCC-1) & + & - & CCR 1 \\
\hline CCL21 (SLC) & + & - & CCR7 \\
\hline
\end{tabular}

*Although IGF-IR and Tie-2 are not upregulated in Kaposi sarcoma, they are always expressed on endothelial cells, implying that they are involved in putative autocrine loops.

Cytokines and receptors whose expression is upregulated in Kaposi sarcoma biopsy samples $(q \leq 0.05)$ are shown, grouped according to putative behavior. Expression of genes shown in bold was previously confirmed in Kaposi sarcoma lesions by immunohistochemistry, in situ hybridization or western blotting. endothelial cells from infiltrating blood vessels, and lose the KSHV episome ${ }^{2,14}$ after a few passages. Our method is robust: most of the molecules previously identified as expressed by Kaposi sarcoma spindle cells in culture and by immunostaining of lesions were present in the Kaposi sarcoma expression signature (Table 1).

The Kaposi sarcoma transcriptome shows that expression of TGF $\beta 1$, TGF $\beta 3$, PDGFA and PDGFB was higher in Kaposi sarcoma than in normal skin $(q \leq 0.05)$. The mRNA levels of their receptors (TGF $\beta R$ and PDGFR $\beta$ ) were also upregulated, indicative of autocrine loops for these growth factors. The HIV coreceptor CCR5 and its ligands CCL5 and CCL8 were upregulated in Kaposi sarcoma, again suggestive of autocrine loops. In contrast, cytokines previously shown to be mitogens for Kaposi sarcoma spindle cells in vitro, including TNF $\alpha$, IL-2, IL-6, VEGF-C/D and oncostatin $\mathrm{M}^{15,16}$, were not substantially overexpressed in Kaposi sarcoma, although their corresponding receptors were, implying that these cytokines might promote Kaposi sarcoma through paracrine effects (Table 1).

We used the Kaposi sarcoma expression signature to compare the Kaposi sarcoma samples with all other samples by calculating average linkage distances between sample groups. A multidimensional scaling (MDS) plot represents these relationships (Fig. 2a). The Kaposi sarcoma group is more closely related to the endothelial cell group than to any other cell type that we tested (Supplementary Table 2 online). As microvascular endothelial cells (MVECs) represent a mixed population of LECs and BECs ${ }^{17}$, we next determined whether the gene expression profile of Kaposi sarcoma is closer to that of LECs or to that of BECs. This analysis showed that Kaposi sarcoma was more similar to LECs than to BECs, using both the Kaposi sarcoma expression signature and a selection of 114 genes that discriminate between LECs and BECs ( $q \leq 0.2$; Fig. $2 \mathbf{b}$ and Supplementary Table $\mathbf{1}$ online). This was confirmed by investigating the number of genes that discriminate between LECs and BECs that are also present in the Kaposi sarcoma expression signature. Substantially more LEC markers (those with higher expression in LECs than in BECs) than BEC markers were present in the Kaposi sarcoma expression signature (Fig. 2c).

Staining for CD31 confirmed that the LECs and BECs were pure populations of endothelial cells, and staining for LEC markers podoplanin and CD206 (ref. 17) confirmed that we isolated pure populations of LECs and BECs. The LEC marker CD206, identified in the Kaposi sarcoma expression signature, was expressed in all Kaposi sarcoma spindle cells (Fig. 2d).

We further explored the close relationship between Kaposi sarcoma and endothelial cells, especially LECs, by testing the susceptibility of several candidate cells, from which Kaposi sarcoma spindle cells have been proposed to originate, to KSHV infection. MVECs were the most susceptible to infection, as determined by flow cytometry for green fluorescent protein (GFP)-tagged KSHV ${ }^{18}$ and quantitative PCR (qPCR) for KSHV genomes (Fig. 3a). The typical nuclear stippling staining pattern of KSHV latent nuclear antigen (LANA) was observed in all GFP-positive cells. Some GFP-negative cells also stained positive for LANA, because the recombinant virus preparation that we used contains wildtype, GFP-negative $\mathrm{KSHV}^{18}$. Next we tested the susceptibility of pure populations of LECs and BECs to KSHV infection. LANA immunofluorescence assays showed that similar numbers of LECs and BECs were infected at various multiplicities of infection (MOIs), with a linear relationship between MOI and number of infected cells $\left(\mathrm{R}^{2}=0.995\right.$ for LECs and $\mathrm{R}^{2}=0.9846$ for BECs). But $\mathrm{qPCR}$ showed that KSHV genomes had a higher copy number in LECs than in BECs (Fig. $3 \mathbf{b}$ and Supplementary Fig. 2 online). Improved KSHV replication in LECs, more efficient maintenance of episomal DNA or a higher density of cellular receptors allowing viral entry on LECs could contribute to this. 
The finding that both LEC and BEC markers are present in the Kaposi sarcoma expression signature (Fig. 2c) indicates that Kaposi sarcoma spindle cells do not faithfully represent either cell lineage, which could be a consequence of KSHV infection. We used GEM analysis to examine the effects of KSHV infection on LEC and BEC transcriptomes. We used the 114 genes that differentiate LECs and BECs in calculating average linkage distances between groups of infected and uninfected LECs and BECs. The genetic profile of KSHV-infected BECs was closer to that of LECs than was the profile of uninfected BECs, and vice versa. These results imply that KSHV infection induces a transcriptional reprogramming of both infected LECs and infected BECs to move away from the uninfected populations and toward each other (Fig. 4a).

We investigated the mechanism of KSHV-induced transcriptional reprogramming by analyzing the expression levels of PROX1. PROX1 is a key LEC-specific homeobox transcription factor that is pivotal in regulating the differentiated LEC and BEC phenotypes by inducing LEC markers and suppressing BEC markers ${ }^{19}$. PROX1 was moderately upregulated in Kaposi sarcoma relative to skin (Fig. $\mathbf{4 b}$; $q=0.155$ ), although the rich lymphatic network of normal skin could dilute significance. PROX1 was downregulated in KSHV-infected LECs, as shown by GEM and qRT-PCR analysis (Fig. 4b).
Certain cytokines and their cognate receptors are present in the Kaposi sarcoma expression signature (Fig. $\mathbf{1 b}$ and Table 1), indicative of autocrine growth loops. Angiopoietin-2 (ANG2) was highly expressed in the Kaposi sarcoma signature, and we confirmed its upregulation in both LECs and BECs infected by KSHV (Fig. 5a and Supplementary Fig. 2 online). ANG2 is involved in angio- and lymphangiogenesis ${ }^{20}$ and is also upregulated by KSHV in MVECs ${ }^{21}$. We examined the clinical relevance of this upregulation by measuring plasma levels of ANG2 in different individuals. We tested all plasma samples for vascular endothelial growth factor (VEGF) and for the lymphangiogenic molecule VEGF-D ${ }^{22}$ in parallel. Previous reports on VEGF plasma levels in individuals with acquired immune deficiency syndrome (AIDS) and Kaposi sarcoma are contradictory ${ }^{23,24}$. VEGF binds VEGFR-1 and VEGFR-2, whereas VEGF-D is a high-affinity ligand and activator of VEGFR-2 and VEGFR-3 and stimulates lymphangiogenesis ${ }^{9}$. VEGFR-3 was present in the Kaposi sarcoma expression signature.

ANG2 levels were significantly higher in individuals with AIDS and Kaposi sarcoma than in any other group $(P=0.003)$. VEGF-D levels were significantly higher in individuals with AIDS and Kaposi sarcoma than in healthy blood donors $(P=0.017)$. VEGF levels were
Figure 2 Relationships between the Kaposi sarcoma expression signature and cell groups. (a) Two-dimensional MDS plot using the Kaposi sarcoma expression signature $(n=1,482$; $q \leq 0.05$ ). Each spot represents a single sample. The average linkage distance between Kaposi sarcoma (KS) and each group is shown (right). The confidence limits shown represent the standard error (mean of intergroup linkages divided by root of the number of linkages). $E C(L+B), L E C s+B E C s$. (b) An MDS plot using the LEC-BEC discriminatory gene signature $(n=114 ; q \leq 0.2)$. The average linkage distance between Kaposi sarcoma (KS) and each group is shown (right). (c) Heat map of genes in the Kaposi sarcoma expression signature that differentiate LECs and BECs. (d) Immunostaining. Left two columns, immunofluorescence on pure LECs and BECs. Both populations stained for CD31, and LECs stained for podoplanin and for CD206. Nuclei were counterstained with Hoechst 33258. Right three columns, immunostaining of tissues showed that CD31 was expressed in lymphatic and blood vessels in lymph nodes and in spindle cells in plaque stage and nodular Kaposi sarcoma lesions. CD206 stained lymphatic vessels (red arrows), but not blood vessels (black arrow) in lymph nodes, and all spindle cells in Kaposi sarcoma. Spindle cell expansion in dermis is indicated (blue arrow). H\&E, hematoxylin and eosin. a

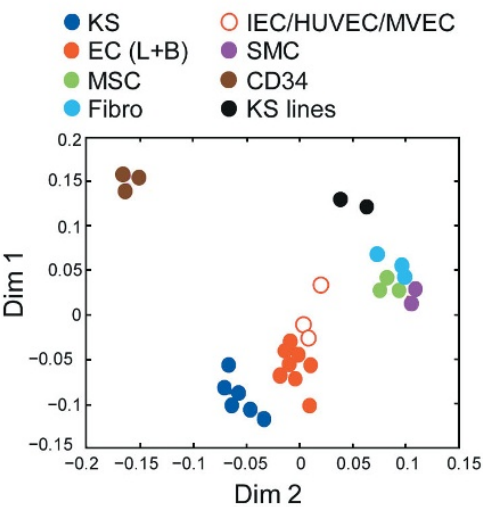

b

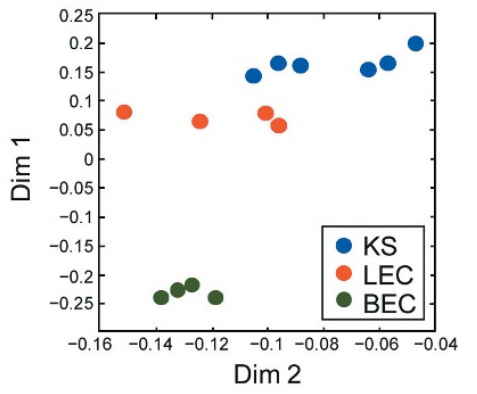

d

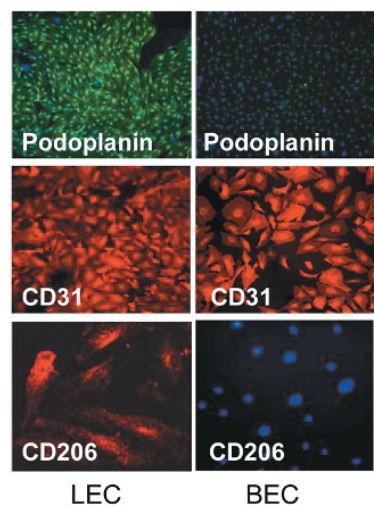

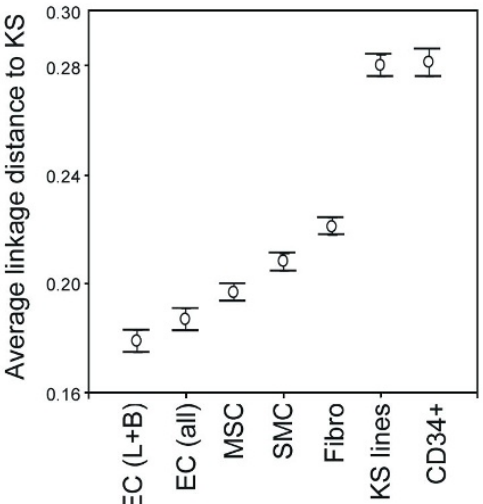

C
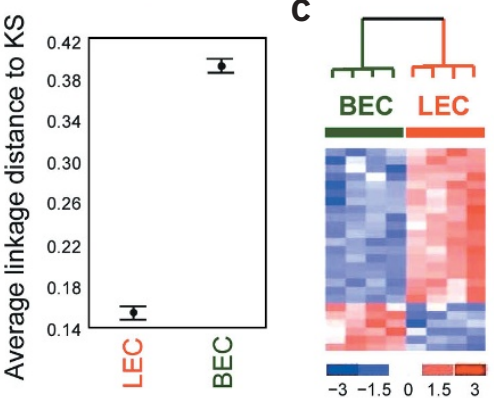

$\begin{array}{lllll}-3 & -1.5 & 0 & 1.5 & 3\end{array}$

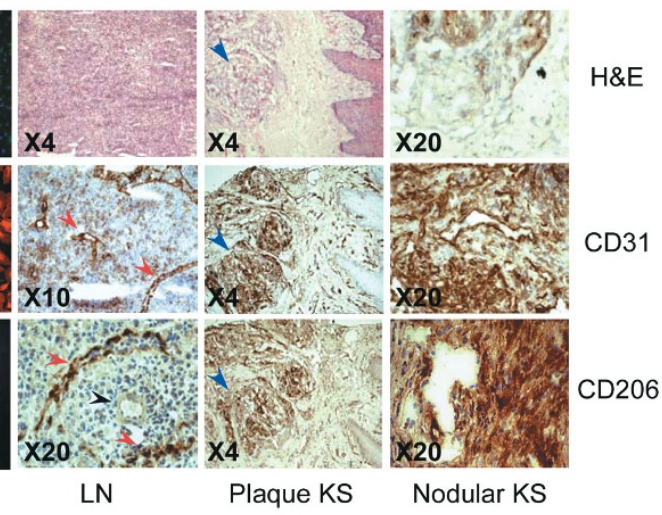


Figure 3 Tropism of KSHV. (a) Quantification of KSHV infection in different cell types. MVECs, mesenchymal stem cells (MSCs), smooth muscle cells (SMCs) and fibroblasts (Fibro) were infected with a recombinant KSHV expressing GFP 18 at similar $\mathrm{MOI}$ and culture conditions. Left, the percentage of GFP-positive cells. Middle, the copy number of KSHV genomes in each infected cell type as determined by qPCR. Right, KSHV LANA staining on infected MVECs shows the nuclear stippling pattern, and red arrows indicate cells also expressing GFP. (b) Quantification of KSHV infection of LECs and BECs. Upper left panel, the percentage of LANA-positive cells in infected LEC and BEC populations was comparable. LECs and BECs from the same donor were infected with GFP-KSHV at different MOIs and LANA immunofluorescence analyzed. Lower left panel, the copy number of KSHV genomes in 100,000 total cells at each MOI was determined by qPCR for LANA. Right, the KSHV genome copies per infected LEC or $\mathrm{BEC}$, at different MOls, after normalization for the number of cells infected (LANA-positive). This is done by dividing genome copies (lower left panel) by 100,000 and then by the percentage of LANA-positive cells (upper left panel).

significantly higher in individuals with HIV than in healthy blood donors $(P=0.012)$. Individuals with AIDS and Kaposi sarcoma also had significantly higher levels of VEGF than healthy blood donors $(P=0.009)$, but this was not significantly different from the other groups (Fig. 5b). VEGF-D levels also correlated with number of Kaposi sarcoma lesions $(P=0.030$; Fig. $5 c$ ). Furthermore, plasma levels of both VEGF-D $(P=0.030)$ and ANG2 $(P=0.041)$ were significantly lower during resolution of Kaposi sarcoma with antiretroviral therapy (ART; Fig. 5d).

$\mathrm{KSHV}$ is the first human pathogen capable of reprogramming endothelial cell transcriptomes. Endothelial cells undergo transdifferentiation in vivo (e.g., venous arterialization), which can be reproduced in vitro by regulating specific genes or culture conditions $^{25}$. Downregulation of PROX1 after infection of LECs may be partly responsible for the genetic drift of infected LECs toward BECs (Fig. 4b). We did not observe upregulation of PROX1 after

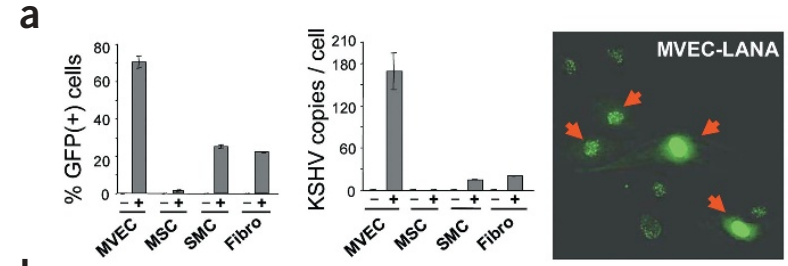

b

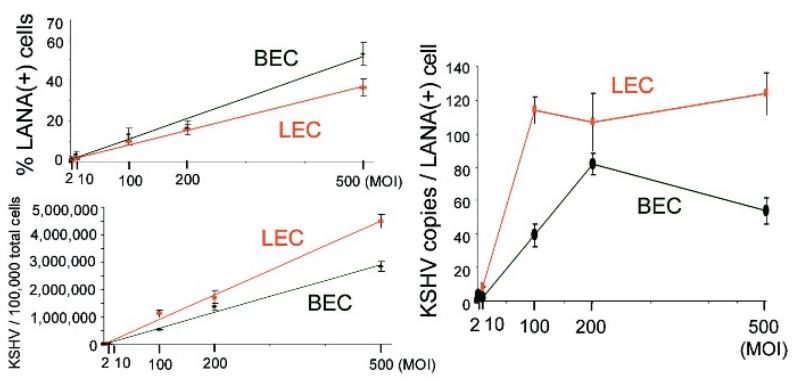

$\mathrm{KSHV}$ infection of $\mathrm{BECs}^{26}$, and this could be a result of using different culture conditions. But the lack of PROX1 induction indicates that other cellular or viral genes are also involved in BEC-to-LEC reprogramming.

We propose two interpretations of our findings. First, KSHV can infect both LECs and BECs in vivo and drives the gene expression profile of each type closer to that of the other. This may explain why, although Kaposi sarcoma spindle cells express mainly LEC markers, BEC markers are also expressed. LECs could be the favored target of infection, however, because more KSHV genome copies per infected cell indicate that LECs are more competent hosts to KSHV in vitro; KSHV is not present in resident blood vessels (BECs) of Kaposi sarcoma lesions ${ }^{8}$ despite the production of KSHV virions in such lesions ${ }^{27}$; and Kaposi sarcoma occurs most frequently at sites rich in LECs (i.e., skin, lymph nodes and mucosa), but not in organs without lymphatic vessels (e.g., brain). A second interpretation that cannot

\section{a}

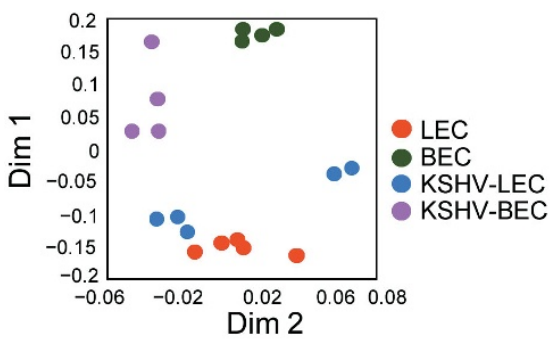

b

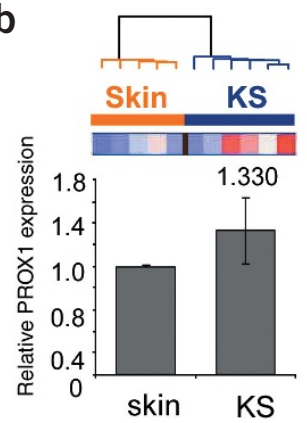

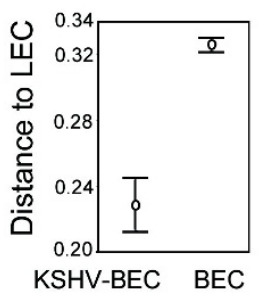

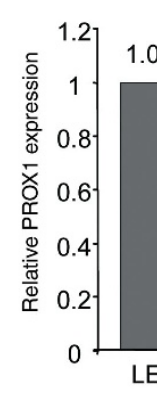

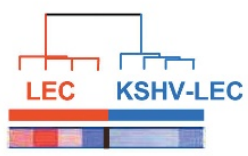

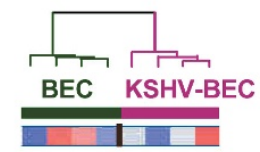

Figure 4 Reprogramming of LECs and BECs by KSHV. (a) Two-dimensional MDS plot of infected and uninfected LECs and BECs using the LECBEC discriminatory gene signature $(n=114$; $q \leq 0.2$ ). Each spot represents a single sample. The average linkage distances between uninfected LECs or BECs and each group are also shown (right). The confidence limits shown represent the standard error (the mean of intergroup linkages divided by root of the number of linkages). The infected LEC and BEC samples seemed to move toward each other, away from the uninfected populations. (b) PROX1 expression levels in Kaposi sarcoma biopsy samples and in KSHV-infected LECs and BECs. GEM data are illustrated for PROX1 in Kaposi sarcoma compared with normal skin and in infected BECs and LECs ( 2 and $7 \mathrm{~d}$ after infection) compared with noninfected ones. PROX1 was upregulated (red) in Kaposi sarcoma and downregulated (blue) in infected LECs but not significantly different after infection of BECs. Histograms show the qRTPCR confirmation of PROX1 levels in two independent experiments (error bars). Relative expressions are normalized to that of skin or to that of uninfected LECs and are indicated in the histogram. 
Figure 5 ELISA of ANG2, VEGF and VEGF-D levels. (a) GEM data are illustrated for ANG2 in Kaposi sarcoma (KS) compared with normal skin, and after infection of BECs and LECs. ANG2 is significantly upregulated (red) in all three heat maps. ELISA confirmation of ANG2 levels in supernatants from infected and uninfected cells. (b) Box plots of VEGF-D, VEGF and ANG2 plasma levels from healthy blood donors (HBD) and three groups of ART-naive individuals infected with HIV-1: individuals with AIDS and Kaposi sarcoma (AIDS-KS), HIV-1-infected individuals seronegative for KSHV (HIV) and HIV-1-infected individuals seropositive for KSHV (HIV/KSHV). Green circles represent outliers. ${ }^{*} P<0.05$ versus healthy blood donors; ${ }^{* *} P<0.05$ versus all other groups. (c) Only VEGF-D was significantly $\left({ }^{*} P<0.05\right)$ elevated in individuals with $>20$ lesions (d) ANG2 and VEGF-D levels correlate with resolution of Kaposi sarcoma lesions during ART. VEGF-D and ANG2 levels decreased significantly $\left({ }^{*} P<0.05\right)$ during ART. VEGF-D and ANG2 levels of nonresponders remained stable, increased or decreased, but remained outside the range of normal healthy blood donors. CR, complete responder; PR, partial responder; NR, nonresponder.

currently be addressed is that KSHV preferentially infects endothelial cell precursors in vivo and drives these cells toward a LEC genotype ${ }^{6}$.

The transcriptional drift of both LECs and BECs infected with KSHV indicates that Kaposi sarcoma spindle cells do not faithfully represent either cell lineage. As lymphangiogenic molecules are elevated in plasma of individuals with AIDS and Kaposi sarcoma, Kaposi sarcoma could be an ideal neoplasm in which to test the efficacy of new antilymphangiogenic therapies.

\section{METHODS}

Cells and media. We grew a GFP-recombinant, KSHV-positive, EBV-negative BCBL-1 cell line ${ }^{18}$ in RPMI medium (Gibco/BRL) supplemented with $20 \%$ heat-inactivated fetal calf serum at a density of $5 \times 10^{5}$ to $1 \times 10^{6}$ cells per ml. We grew an AIDS Kaposi sarcoma cell line, KS Y-1, and an iatrogenic Kaposi sarcoma cell line, KS-IMM, in Dulbecco's modified Eagle medium (Gibco/BRL) supplemented with $5 \%$ heat-inactivated fetal calf serum. We cultured primary MVECs (Clonetics) and human umbilical vein endothelial cells (Clonetics) in EGM-2 MV BulletKit medium (Clonetics). We isolated human LECs and BECs using a polyclonal podoplanin antibody (provided by D. Kerjaschki; University of Vienna, Austria) from three different batches of MVECs from three different donors and cultured them in Endothelial Cell Growth Medium MV (PromoCell) plus $10 \mathrm{ng} \mathrm{m}^{-1}$ of VEGF-C as described ${ }^{17}$. Immortalized human endothelial cells were provided by M.J. O'Hare (University College of London) and cultured in EGM-2 MV BulletKit medium. We cultured human primary uterine and aortic smooth muscle cells (Clonetics) in SmGM-3 BulletKit medium (Clonetics). We cultured primary human dermal fibroblasts (Clonetics) and isolated two primary fibroblasts (CR1E and SS1MB) from two different donors and cultured them in FGM-2 MV BulletKit medium (Clonetics). We isolated human bone marrow CD34 mesenchymal stem cells and $\mathrm{CD} 34^{+}$stem cells from different donors. CD $34^{+}$ cells were directly used for RNA extraction but mesenchymal stem cells were further cultured in MesenCult basal medium (StemCell) supplemented with mesenchymal stem cell stimulatory supplements (StemCell).

Virus and infection. We used a recombinant KSHV expressing GFP produced from BCBL-1 cells after induction of the lytic cycle with $20 \mathrm{ng} \mathrm{ml}^{-1}$ of tetradecanoyl phorbol acetate (Sigma) for $4-5 \mathrm{~d}$ (ref. 18). We omitted polybrene from the infection to exclude non-receptor-mediated, nonspecific viral entry. For most infections, we also measured KSHV copy numbers in cells by qPCR. Unless otherwise specified, all infections were done at an MOI of 250 and in triplicate. We determined the number of cells expressing GFP by flow cytometry in triplicate $2 \mathrm{~d}$ after infection.

Tissue samples and array processing. We assembled a collection of 24 cell cultures, five AIDS-associated Kaposi sarcomas from five different individuals, one classic Kaposi sarcoma and five skin biopsy samples from five different individuals. All Kaposi sarcoma biopsy samples used for the array studies were nodular lesions that contained $>80 \%$ spindle cells. Skin samples and Kaposi sarcoma
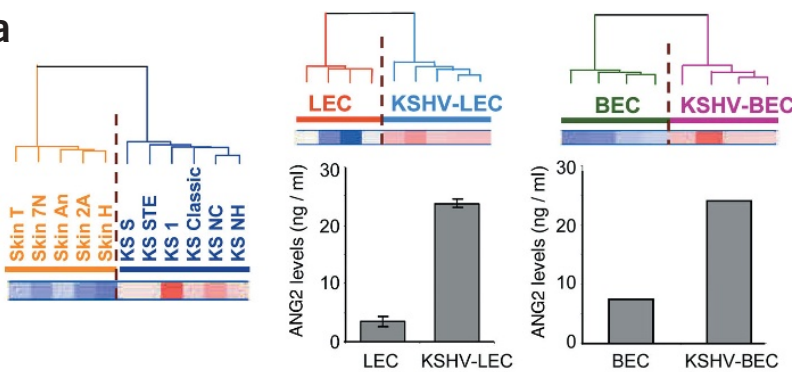

b

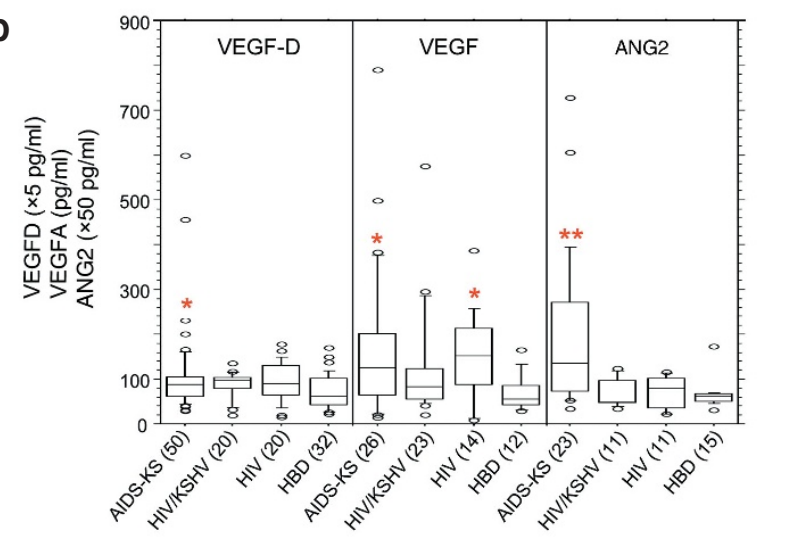

C
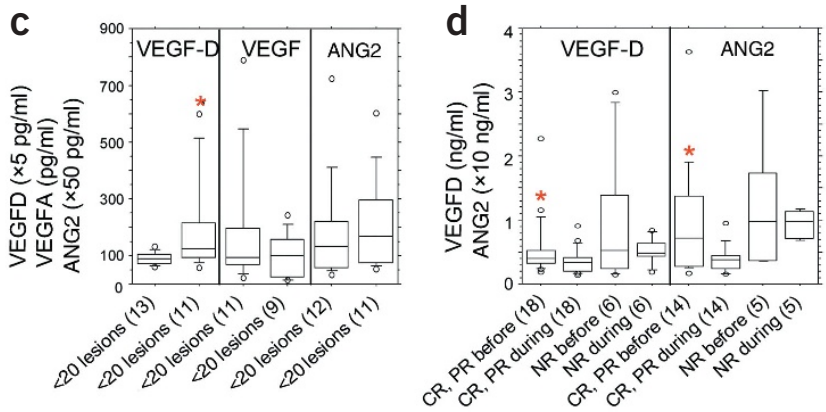

biopsy samples were frozen fresh in liquid nitrogen and stored at $-70{ }^{\circ} \mathrm{C}$. All experiments used Affymetrix Hg-U133A oligonucleotide arrays containing 22,283 human gene probes. We used total RNA from each sample to prepare biotinylated target RNA, with minor modifications from the manufacturer's recommendations. We first homogenized clinical samples and extracted the RNA using Trizol solution (Gibco/BRL) followed by RNeasy Mini kit (Qiagen) purification. We also collected total RNA from culture cells using the RNeasy Mini kit and assessed RNA integrity and quantity using RNA 6000 Nano chips (Agilent). We used $5 \mu \mathrm{g}$ of total RNA to generate first-strand cDNA using a T7-linked oligo(dT) primer and the Custom SuperScript dscDNA Synthesis Kit (Invitrogen). After second-strand synthesis, we carried out in vitro transcription with biotinylated UTP and CTP using the Enzo BioArray HighYield RNA Transcript Labeling Kit (Affymetrix) and the GeneChip Sample Cleanup Module (Affymetrix), resulting in 100-fold amplification of RNA. We processed $20 \mu \mathrm{g}$ of the synthesized biotinylated cRNA probes according to the manufacturer's recommendation using an Affymetrix GeneChip Instrument System (see URL below). We added spike controls to fragmented cRNA before overnight hybridization. We then washed arrays and stained them with streptavidin-phycoerythrin, before scanning them on an Affymetrix GeneChip scanner. After scanning, we visually assessed array images to confirm scanner alignment and the absence of marked bubbles or scratches on the chip surface. We confirmed that $3^{\prime} / 5^{\prime}$ ratios for GAPDH and $\beta$-actin were within acceptable limits and found that BioB spike controls were present on all chips, with $\mathrm{BioC}, \mathrm{BioD}$ and $\mathrm{CreX}$ also present in increasing intensity. When scaled to a target intensity of 100 (using Affymetrix Microarray Suite software MAS v5.1), scaling factors for all arrays were within acceptable limits, as were background, Q values and mean intensities. Details of quality control measures can be found in the ArrayExpress database (see URL and accession numbers below). 
Computational analysis. We calculated RMA log expression units ${ }^{28}$ from Affymetrix GeneChip array data using the 'affy' package of the Bioconductor suite of software for the R statistical programming language (see URLs below). The default RMA settings were used to background-correct, normalize and summarize all expression values. We identified significant differences between sample groups using a previously described method ${ }^{29}$. We calculated a t-statistic as normal for each gene and then calculated a $P$ value using a modified permutation test as described ${ }^{29}$. To control the multiple testing error, we then applied a false discovery rate algorithm to these $P$ values to calculate a set of $q$ values, thresholds of the expected proportion of false positives, or false rejections of the null hypothesis ${ }^{29}$. For example, calling the differential expression of a gene significant with a $q$ value of 0.05 assures that fewer than $5 \%$ of genes with $q$ values less than 0.05 can be expected to be false positives.

We created heat maps of differential gene expression (Figs. $\mathbf{1 b}$ and $\mathbf{2 c}$ ) by exporting the selected RMA data to the DChip microarray analysis package ${ }^{13}$. We carried out classical MDS ${ }^{30}$ using the standard function of the Matlab statistical software package (Mathworks); this reflects how the various sample groups are related. We used the Pearson correlation subtracted from unity to measure the distance between samples, providing bounded distances in the range $(0,2)$. We calculated the distance between two groups of samples using the average linkage measure (the mean of all pairwise distances (linkages) between members of the two groups concerned). The standard error of the average linkage distance between two groups (the standard deviation of pairwise linkages divided by the square root of the number of linkages) is quoted when intergroup distances are compared in the text.

Immunofluorescence and immunostaining. We grew cell preparations on coverslips, fixed them with $4 \%$ paraformaldehyde (SERVA Electrophoresis) and permeabilized them with $0.2 \%$ Triton X100 (Sigma). We blocked cells for 30 min with phosphate-buffered saline and $1 \%$ bovine serum albumin factor $\mathrm{V}$ (Sigma). For KSHV LANA, we used the monoclonal antibodies LN53 at a 1:500 dilution. We stained cultivated endothelial cells with monoclonal antibody to CD206 (PharMingen; 1:100) and CD31 (Sigma; 1:200). All primary and secondary antibodies were incubated with the cells for $30 \mathrm{~min}$ at $37^{\circ} \mathrm{C}$. We used rabbit antibody to rat IgGs conjugated to fluorescein isothiocyanate (1:120, DakoCytomation) or goat $\mathrm{F}(\mathrm{ab})^{\prime} 2$ fragment to mouse IgGs conjugated to fluorescein isothiocyanate or R-phycoerythrin (1:200, DakoCytomation) as secondary antibodies. We counterstained cell nuclei with Hoechst 33258 (Sigma) and examined them by fluorescence confocal microscopy (Leica).

For immunostaining of tissues, we used monoclonal antibodies to CD31 (Dakocytomation) and to CD206 (Autogenbioclear). We cut fresh-frozen sections at $5-\mu \mathrm{m}$ intervals, picked them up onto Vectabond-coated slides, wrapped them in foil and placed them at $-20{ }^{\circ} \mathrm{C}$. We carried out antigen retrieval by placing the fixed sections in boiling citrate buffer $(\mathrm{pH}$ 6.0) in the microwave for $2 \mathrm{~min}$, cooling them in water and transferring them to Ventana APK wash solution. The antibodies were used in accordance with the manufacturers' instructions. We carried out immunohistochemical analysis using the Ventana neXESimmunocytochemistry staining system.

Real-time qPCR and RT-PCR. We treated cell culture supernatants (400 $\mu \mathrm{l})$ with $20 \mathrm{U} \mathrm{ml}^{-1}$ of RNase-free DNase (DNA-free, Ambion) at $37^{\circ} \mathrm{C}$ for $1 \mathrm{~h}$ to degrade any free KSHV DNA. We extracted DNA from supernatants or cell pellets using QIAamp blood Mini kit (Qiagen) according to the manufacturer's protocol. We determined KSHV genome copy number after adjustment to the number of cells analyzed (two copies of GAPDH per cell). A fragment of DNA containing part of the genes encoding GAPDH and LANA was previously developed and used as a standard in the assay. We detected and analyzed specific products using a Perkin-Elmer 7700 sequence detector (PE Applied Biosystems). We carried out real-time PCR using the SYBR Green PCR Master Mix (Applied Biosystems) in duplicate, with triplicate nontemplate controls in a $25-\mu \mathrm{l}$ PCR reaction. We used $1 \mu \mathrm{l}$ of cDNA in a $25-\mu \mathrm{l}$ PCR mixture containing $1 \times$ SYBR Green PCR mix (Applied Biosystems), $0.3 \mu \mathrm{M}$ of the PROX1 forward and reverse primers or $0.3 \mu \mathrm{M}$ of the GAPDH forward and reverse primers. We then amplified the cDNAs by denaturation for 10 min at $95^{\circ} \mathrm{C}$, followed by 40 cycles of denaturation at $95^{\circ} \mathrm{C}$ for $15 \mathrm{~s}$ and annealing-extension at $60^{\circ} \mathrm{C}$ for $1 \mathrm{~min}$. Primer optimization and efficiency were done before the relative quantification of the expression of the gene (data not shown). We determined the relative expression of PROX1 on the basis of the $C_{\mathrm{T}}$ value (the PCR cycle at which an increase in reporter fluorescence above a baseline signal can first be detected) using the $\Delta \Delta \mathrm{Ct}$ method. Primer and probe sequences are available on request.

Determination of cytokine levels. We collected culture supernatants from infected and uninfected cells 2, 5 and $7 \mathrm{~d}$ after infection from LECs, and $7 \mathrm{~d}$ after infection from BECs. We took plasma samples from individuals infected with HIV-1 from University College London Hospitals. We obtained approval from Camden and Islington Community Local Research Ethics Committee. We assessed KSHV serostatus by immunofluorescence analysis for antibodies to LANA. Initially, we classified samples into three categories: individuals with AIDS and Kaposi sarcoma, KSHV-seropositive individuals without Kaposi sarcoma and KSHV-seronegative individuals without Kaposi sarcoma. Cytokine levels of individuals in these groups were compared with those of healthy blood donors. All groups were age-matched, and HIV-1-positive groups had not undergone ART and were matched with respect to $\mathrm{CD} 4^{+} \mathrm{T}$-cell count. Only fresh-frozen plasma samples were used for the study. The size of group studied varied between cytokine studies (e.g., for AIDS and Kaposi sarcoma, $n=50$ for VEGF-D and $n=23$ for ANG2) because multiple samples were not available from all individuals. For 24 individuals with AIDS and Kaposi sarcoma, plasma samples were also available during ART. We obtained informed consent from all the enrolled individuals to take blood samples and approval from the Ethics Committee of Camden \& Islington Community Health Services NHS Trust.

We determined VEGF, VEGF-D and ANG2 levels with commercially available quantitative ELISA kits (R\&D Systems). We tested all plasma samples in duplicate according to the manufacturer's instructions. We constructed a standard curve between concentration and absorbance at $450 \mathrm{~nm}\left(\mathrm{~A}_{450}\right)$ each time with the use of recombinant human protein standards. We confirmed minimum interassay variability by repeated testing of identical plasma samples and recombinant protein controls. We compared cytokine levels of different groups using the parametric, two-tailed, unpaired Student's $t$-test. We used the same test to assess the difference between the group of individuals with AIDS and Kaposi sarcoma with more than 20 lesions and those with fewer than 20 lesions. The effect of ART on VEGF-D and ANG2 levels was assessed using the parametric, two-tailed, paired Student's $t$-test.

In box plots, the box indicates the lower and upper quartiles, whereas the line in the box is the median of each data set. The points at the end of the lines outside the box represent the minimum and maximum of the data set. Circles represent outliers. Graphs were drawn using the StatView statistical software package (SAS Institute).

URLs. Information on Affymetrix $\mathrm{Hg}-\mathrm{U} 133 \mathrm{~A}$ oligonucleotide arrays is available at http://www.affymetrix.com/products/arrays/specific/hgu133.affx. Information on biotinylated target RNA preparation is available at http:// www.affymetrix.com/support/technical/manual/expression_manual.affx. The Affymetrix GeneChip instrument system is described at http://www. affymetrix.com/support/downloads/manuals/expression_s2_manual.pdf. The ArrayExpress database is available at http://www.ebi.ac.uk/arrayexpress. The Bioconductor suite of software is available at http://www.bioconductor.org. Information on the $\mathrm{R}$ statistical programming language is available at http://www.r-project.org.

Accession numbers. Details of quality control measures and all microarray data can be found in the ArrayExpress database with the accession number E-MEXP-66.

\section{Note: Supplementary information is available on the Nature Genetics website.}

\section{ACKNOWLEDGMENTS}

We thank the AIDS-Malignancy Bank of the US National Cancer Institute for providing the Kaposi sarcoma biopsy samples used in this study; M. Byers, S. Crane, D. MacDonald, D.B. Guiliano, A. Godfrey and R. Dunbar for technical expertise; J. Vieira for providing the BCBL-1 cell line producing KSHV-GFP; and R. Ambinder for providing the JSC-1 cell line. We apologize to the many scientists whose work we did not cite due to space constraints. This work is supported by Wellcome Trust, European Union, Cancer Research UK and the Medical Research Council. 
COMPETING INTERESTS STATEMENT

The authors declare that they have no competing financial interests.

Received 27 April; accepted 28 May 2004

Published online at http://www.nature.com/naturegenetics/

1. Regezi, J.A. et al. Human immunodeficiency virus-associated oral Kaposi's sarcoma. A heterogeneous cell population dominated by spindle-shaped endothelial cells. Am. J. Pathol. 143, 240-249 (1993).

2. Kaaya, E.E. et al. Heterogeneity of spindle cells in Kaposi's sarcoma: comparison of cells in lesions and in culture. J. Acquir. Immune Defic. Syndr. Hum. Retrovirol. 10 295-305 (1995).

3. Gallo, R.C. The enigmas of Kaposi's sarcoma. Science 282, 1837-1839 (1998).

4. Kahn, H.J., Bailey, D. \& Marks, A. Monoclonal antibody D2-40, a new marker of lymphatic endothelium, reacts with Kaposi's sarcoma and a subset of angiosarcomas. Mod. Pathol. 15, 434-440 (2002).

5. Chang, Y. et al. Identification of herpesvirus-like DNA sequences in AIDS-associated Kaposi's sarcoma. Science 266, 1865-1869 (1994).

6. Boshoff, C. \& Weiss, R. AIDS-related malignancies. Nat. Rev. Cancer 2, 373-382 (2002).

7. Ganem, D. KSHV and Kaposi's sarcoma: the end of the beginning? Cel/ 91, 157-160 (1997).

8. Dupin, N. et al. Distribution of human herpesvirus-8 latently infected cells in Kaposi's sarcoma, multicentric Castleman's disease, and primary effusion lymphoma. Proc. Natl. Acad. Sci. USA 96, 4546-4551 (1999).

9. Stacker, S.A., Achen, M.G., Jussila, L., Baldwin, M.E. \& Alitalo, K. Lymphangiogenesis and cancer metastasis. Nat. Rev. Cancer 2, 573-583 (2002).

10. Flore, O. et al. Transformation of primary human endothelial cells by Kaposi's sarcomaassociated herpesvirus. Nature 394, 588-592 (1998)

11. Moses, A.V. et al. Long-term infection and transformation of dermal microvascular endothelial. J. Virol. 73, 6892-6902 (1999).

12. Ciufo, D.M. et al. Spindle cell conversion by Kaposi's sarcoma-associated herpesvirus: formation of colonies and plaques with mixed lytic and latent gene expression in infected primary dermal microvascular endothelial cell cultures. J. Virol. 75, 5614-5626 (2001).

13. Li, C. \& Wong, W.H. Model-based analysis of oligonucleotide arrays: expression index computation and outlier detection. Proc. NatI. Acad. Sci. USA 98, 31-36 (2001).

14. Lebbe, C. et al. Characterization of in vitro culture of HIV-negative Kaposi's sarcomaderived cells. In vitro responses to alfa interferon. Arch. Dermatol. Res. 289, 421-428 (1997).

15. Samaniego, F., Markham, P.D., Gendelman, R., Gallo, R.C. \& Ensoli, B. Inflammatory cytokines induce endothelial cells to produce and release basic fibroblast growth factor and to promote Kaposi's sarcoma-like lesions in nude mice. J. Immunol. 158, 1887-1894 (1997).

16. Brockmeyer, N.H. et al. Cytokine profile of HIV-positive Kaposi's sarcoma derived cells in vitro. Eur. J. Med. Res. 4, 95-100 (1999).

17. Makinen, T. et al. Isolated lymphatic endothelial cells transduce growth, survival and migratory signals via the VEGF-C/D receptor VEGFR-3. EMBO J. 20, 4762-4273 (2001).

18. Vieira, J., O'Hearn, P., Kimball, L., Chandran, B. \& Corey, L. Activation of Kaposi's sarcoma-associated herpesvirus (human herpesvirus 8 ) lytic replication by human cytomegalovirus. J. Virol. 75, 1378-1386 (2001).

19. Petrova, T.V. et al. Lymphatic endothelial reprogramming of vascular endothelial cells by the Prox-1 homeobox transcription factor. EMBO J. 21, 4593-4599 (2002).

20. Gale, N.W. et al. Angiopoietin-2 is required for postnatal angiogenesis and lymphatic patterning, and only the latter role is rescued by Angiopoietin-1. Dev. Cel/ 3, 411-423 (2002).

21. Poole, L.J. et al. Altered patterns of cellular gene expression in dermal microvascular endothelial cells infected with Kaposi's sarcoma-associated herpesvirus. J. Virol. 76, 3395-3420 (2002).

22. Ferrara, N., Gerber, H.P. \& LeCouter, J. The biology of VEGF and its receptors. Nat. Med. 9, 669-676 (2003).

23. Ascherl, G. et al. Infection with human immunodeficiency virus-1 increases expression of vascular endothelial cell growth factor in T cells: implications for acquired immunodeficiency syndrome-associated vasculopathy. Blood 93, 4232-4241 (1999).

24. Renwick, N. et al. Vascular endothelial growth factor levels in serum do not increase following HIV type 1 and HHV8 seroconversion and lack correlation with AIDS-related Kaposi's sarcoma. AIDS Res. Hum. Retroviruses 18, 695-698 (2002).

25. Shin, D. et al. Expression of ephrinB2 identifies a stable genetic difference between arterial and venous vascular smooth muscle as well as endothelial cells, and marks subsets of microvessels at sites of adult neovascularization. Dev. Biol. 230, 139-150 (2001).

26. Hong, Y.-K. et al. Lymphatic reprogramming of blood vascular endothelium by Kaposi sarcoma-associated herpesvirus. Nat. Genet. advance online publication, 27 June 2004 (doi:10.1038/ng1383).

27. Orenstein, J.M. et al. Visualization of human herpesvirus type 8 in Kaposi's sarcoma by light and transmission electron microscopy. AIDS 11, F35-F45 (1997).

28. Irizarry, R.A. et al. Summaries of Affymetrix GeneChip probe level data. Nucleic Acids Res. 31, E15 (2003).

29. Storey, J.D. \& Tibshirani, R. Statistical significance for genomewide studies. Proc. Natl. Acad. Sci. USA 100, 9440-9445 (2003).

30. Kruskal, J.B. Multidimensional scaling by optimizing goodness of fit to a nonmetric hypothesis. Psychometrika 29, 1-27 (1964). 